\title{
Side scan Sonar Image Segmentation and Feature Extraction
}

\author{
Miguel Pinto, Bruno Ferreira, Aníbal Matos, Nuno Cruz \\ FEUP-DEEC, Rua Dr. Roberto Frias, 4200-465 Porto, Portugal \\ ee04134@fe.up.pt, ee04018@fe.up.pt, anibal@fe.up.pt, nacruz@fe.up.pt
}

\begin{abstract}
This paper describes an algorithm to make the treatment, segmentation, skeleton and characteristics extraction from acoustic images obtained from a side scan sonar. The fundamental goal is to implement a system that endows a autonomous vehicle with the capacity to know its own distance to the marine bottom and to features located on the marine environment. This features extraction would improve vehicle navigation and allow it to navigate relative to features like an underwater piper on the sea floor or a vertical wall.

This paper was made based on Imagenex SportScan (side scan sonar) whose function is the observation of environment. Also the autonomous surface vehicle (ASV) ZARCO was used to transport the side scan sonar. Both the vehicles belong to The OceansSys Group DEEC-FEUP. A communication interface between the ASV ZARCO, Imagenex SportScan, and a static laptop that allows the observation of sonar data in real time is also described in this paper.

The algorithms and routines implemented were validated with real acoustic images acquired during a mission. Results from algorithms application and features extraction are shown in this paper.
\end{abstract}

\section{INTRODUCTION}

The purpose of this paper is the treatment, segmentation and extraction of features from acoustic images provided by side scan sonar.

Two sensors or methods that provide environment observations are optical vision and sonar. The light absorption to deep waters makes the use of optical vision impossible without artificial light. The absorption of the light increases with the depth, then the use of optical vision requires artificial light [1]. Also when there are suspended particles or dirtiness inside the water, the optical vision is affected and the image acquired has lower quality. Thus, this work is fundamental focused in observations from sea bed obtained by side scan sonar.

Imagine that an AUV (Autonomous Underwater Vehicle) or an ASV (Autonomous Surface Vehicle) navigates based on terrain and the goal is make the AUV follow a reference path, at a reference distance, from a feature with an infinite length. Thus the core of this work is to show a way to segment and extract features that provide the AUV with the capacity to know the relative distance to features and thus navigate relative to them.

The feature considered in this work is a vertical wall. The work developed and described here is based on the Imagenex Sportscan [2], side scan sonar.

Give to the autonomous vehicle (ASV or AUV) the capacity to know its relative localization to features that exists on the marine environment and navigate relative them is the same that give higher degree of autonomy to the vehicle.
So, the real opportunity of this paper and the work developed was the true definition of autonomy [3] and [4].

In section sonar data are described. The third section describes image analyses and standard algorithms to image segmentation and characteristics extraction. In section IV the Imagenex SportScan sonar (side scan sonar) is described, while in the fifth section the fundamental characteristics from ASV ZARCO are presented. The sixth section shows the communication interface developed between Imagenex SportScan, Zarco and an laptop. In section VII the operation limits of side scan sonar are studied. In section VIII and IX are shown results from the algorithms application on real acoustic images obtained through sonar acquisition. Finally in section $\mathrm{X}$ project limitations are explained.

\section{SONAR DATA}

The terrain-based navigation [5], based on natural landmarks has essentially two phases:

-Construction Map, knowing the location of the vehicle in the world by other methods of localization.

-Localization of the vehicle based on the world map constructed a priori.

This second step use environment observation like sonar. In the works developed and related by [6 to 10] the navigation based in terrain uses sonar data.

In [7], a $360^{\circ}$ sonar is used to navigation into structured underwater environments.

\section{A. Sonar, SOund Navigation And Range}

Sonar can be used to obtain acoustic images of the sea bed, allowing the extraction an identification of natural landmarks.

The side scan sonar comparative with forward-look sonar gives more quality images from sea bed. Thus it provides better and easily extraction of features [11].

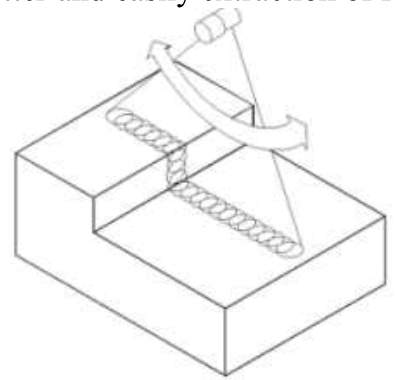

a)

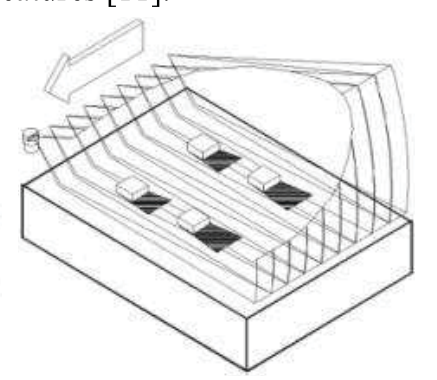

b)
Figure 1 a) Forward-look scan, [2]. b) Side scan, [2].

In both them [11] and [12], the sonar type chosen to development of the work was the side scan sonar. In this work the chosen sonar was also side scan sonar, more precisely, the Imaginex Sportscan, that is shown in Figure 2. 
An image obtained by the acoustic side scan sonar is the echo of lines of force in time, equally spaced between them [13].

Other sonar type is the multibeam sonar. It correspond a sonar type in which the quality of acoustic image obtained is better than side scan or forward-look. The information collected is larger, which allows terrain-based navigation of higher quality.

The multibeam sonar, gives a three-dimensional pictures of the environment. It is composed by an array of sonars (multibeam), arranged in the same direction and angle of incidence fixed, known and that differs between them, [14].

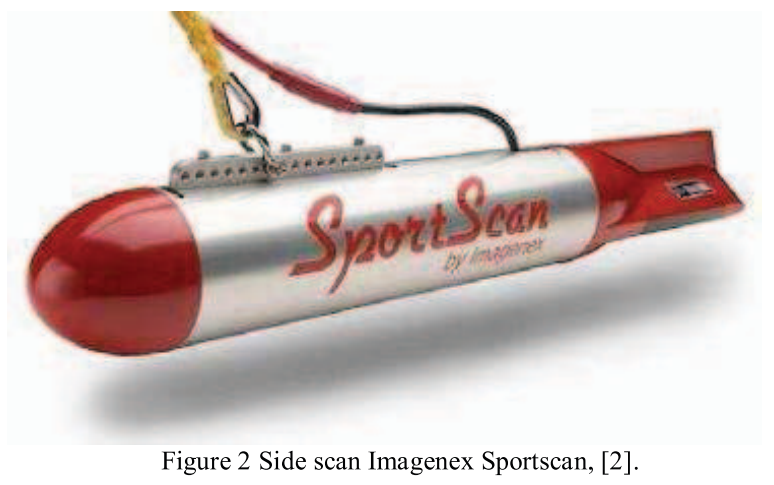

B. Side scan data

A set of data can be identified from the side scan acoustic image Figure 3. The most relevant data are:

-The size of the no-echo zone, $(\mathrm{H})$ allows us to know the height above the floor at which the AUV is.

-A sum of no-echo zone with the area of the bottom echo and the target, $(\mathrm{H}+\mathrm{r})$, allows to know the distance between the submarine and the target.

-A shadow zone of the target (S), where there is no-echo, lets us know the height of the target. This height is given by the expression:

$$
h=\frac{H . S}{r+S}
$$

All these characteristics can be obtained by image processing algorithms.

The algorithms of segmentation and extraction of features should be flexible and then be able to handle images whose quality is lower than the figures $3, a$ ) and $b$ ).

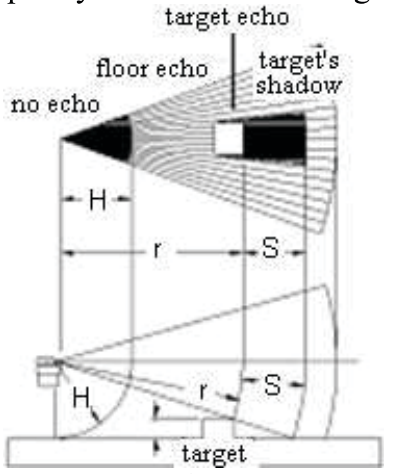

a)

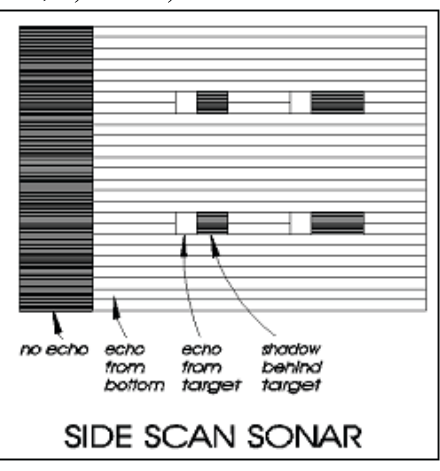

b)
Figure 3 a) Beam Observation, Target values (H, r, h and s), [2]. b) Side scan image, [2].

\section{IMAGE ANALYSIS}

One image is a matrix of pixies where each pixel is function of it own position $(x, y)$ on the image plan. Each pixel is a value of light intensity and thus is function of the incident energy on the image plan. So, a monochromatic image is function $f(x, y)$ where $\mathrm{f}$ is between black and white values, i.e., belongs to the grey scale, charpter "Digital Image Fundamentals" from [15].

On this way, on the manochromatic image the function $f(x, y)$ is inside the gap:

$$
0(\text { Black }) \leq f(x, y) \leq 255(\text { White })
$$

The acoustic image obtained by sonar side scan Imagenex SportScan is a monochromatic image where their pixies are between 0 and 127 gray scale value.

A. Image pre-processing

Sometimes the images obtained have some degradation sources. This degradation can be result from compression, expansion, image rotation or random noise.

So, there are a set of operators that are responsible to image pre-processing and image treatment. There are: punctual, local and global operators.

The local operators have huge importance on image preprocessing since they turn possible the noise elimination or reduction for next step, segmentation. They are described on chapter "Image Enhancement" from [15], with them application can be obtained a result image where each pixel is result from the operation on the original pixel and it vicinity..

Thus, some local operators are used to noise reduction like and are called Smoothing Filters. Other examples are the average, median, dilate, erode, band pass, high pass and low pass filters, chapter "Image Enhancement" from [15].

\section{B. Image post-processing}

After image pre-processing appears the segmentation.

In processes where the artificial vision is used the goal is not obtain an image (with higher quality) from the original, but obtain features information (sometimes numerical information) from the original image. The segmentation divides the image on parts or objects that constitutes it, chapter "Image Segmentation" from [15]. The same happens with sonar and the obtained images from the sea floor.

There are two fundamental categories of segmentation, the discontinuity and similarity. The discontinuity is based on huge variations inside the image (like borders) in the image that is treated. Thus the discontinuity focus essencialy on edge and line detection, chapter "Image Segmentation" on [17].

On the image segmentation the edge detection is fundamental. The edge detection is the separation of the homogeneous zones and variables zones, like borders, in the image.

Examples of diferential operator that turn possible the edge detection are the gradient operators like the Sobel mask $(A=2)$ or Prewitt mask $(A=2)$. These operators are characterized for the next matrix, for horizontal edge detection. 


$$
\text { Horizontal gradient }=\left[\begin{array}{ccc}
-1 & -A & -1 \\
0 & 0 & 0 \\
1 & A & 1
\end{array}\right]
$$

After the edge detection application its necessary obtain the equations from the borders detected, since are these equation that turn possible the distance to bottom and feature determination.

The algorithm that allows the equations determination is called by Hough transform and it is related on chapter "Image Segmentation" from [15].

\section{Image skeleton}

The skeleton operations are used as methods for smoothing or border projections elimination of an object, chapter "Representation and Description" from [15].

Skeleton is based in two fundamental operators: the dilation and the erosion.

When these two operators are sequential applied on the image it is obtained other two important operations: the open and the close.

The open operation is the dilation applied after the erosion and it is useful for smoothing contours of the image and borders of objects. Eliminates projections of the borders on objects and eliminates holes in the bottom of the image.

The application of erosion in the image eliminates the holes from the bottom of the image whose size is smaller than the structuring element window. In the other way increase the size of holes in objects of the image and reducing the size of its borders. It must then apply the operation of dilation with the same structuring element to restore the pixels of the borders. With this operation are eliminated holes inside objects of the image.

The close operation is the erosion applied after the dilation.

This operation is used for holes elimination and gaps in the border of the image objects.

When applied the dilation on the image, the holes inside the objects are removed, since its size is less than the operator of dilation. The borders of objects grow and holes in the bottom of the image increases in size. Thus, it is then applied the erosion which reduces the size of holes in the bottom of the image.

After this operation border gaps and holes inside the objects have been met.

\section{IMAGENEX SPORTSCAN}

The main equipment used to the work developed is the Imagenex SporScan, that is side scan sonar and all acoustic images obtained from river floor and presented here are provided by it.

All work was made with The OceanSys Group FEUPDEEC (Faculty of engineering of the University of Porto Department of Engineering Electronic and Computers) collaboration and thus all equipment used like the Imagenex SportScan belong them [16].

TABLE I

IMAGENEX SPORTSCAN SPECIFICATIONS

\begin{tabular}{|c|c|}
\hline \multicolumn{2}{|c|}{ IMAGENEX SPORTSCAN SPECIFICATIONS. } \\
\hline Frequency mode & $330 \mathrm{kHz} 800 \mathrm{kHz}$ \\
\hline Beam with & One or two sides \\
\hline
\end{tabular}

\begin{tabular}{|c|c|}
\hline & $1.8^{\circ} \times 60^{\circ}$ \\
& $\begin{array}{c}\text { For frequency mode: } 800 \mathrm{kHz}: \\
0.7^{\circ} \times 30^{\circ}\end{array}$ \\
\hline Resolution & $\begin{array}{c}\text { Both sides displayed: } \\
\text { Range scale } \div 250 \\
\end{array}$ \\
Single side displayed: Range scale $\div$ \\
500
\end{tabular}

This is portable sonar and some fundamental physical and hardware specifications from this sonar is shown with Table 1 help.

It is small sonar with application on search and exploration AUVs or ASVs. It has $114 \mathrm{~mm}$ of diameter and $833 \mathrm{~mm}$ of length. It weight about $4.5 \mathrm{~kg}$ inside the water.

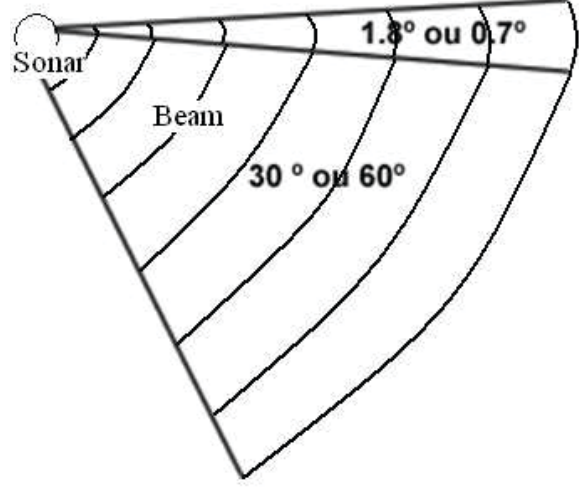

Figure 4 Acoustic beam from sonar with two configurable incidence angles for high and low frequency modes (can be seen on table 1) .

Others fundamental characteristics of Imagenex SportScan are:

-It needs a RS-232 command of configuration or request to data, with 115200 Bps of data exchange speed.

- The request is a message of 27 bytes length.

- Sonar response frame length is 513 bytes, where 13 bytes from them are debug bytes (header bytes) and the next 500 bytes are data of echo strength.

The request frame for sonar data is $B_{\text {req }}$ bytes long. The sonar response length is $B_{\text {res }}$. The acquisition time of data is equal to the time between acoustic impulse transmission and the respective echo arrivals. Considering the data exchange between the vehicle and sonar based on RS-232, the total time acquisition of a slice is given by:

$$
T=B R \cdot B i t s\left(B_{\text {req }}+B_{\text {res }}\right)+2 \frac{\text { Range }}{v},
$$

where $B R$ is de baud rate, Bits the number bits per byte, Range the sonar reach in meters and $v$ is the velocity of propagation of the sound in water.

Thus, for a request frame with 27 bytes long, sent with 115.2 Kbps of baud rate, the request time comes equal to:

$$
\text { Trequest }=\frac{27 \cdot 10}{115200} \cong 2,3 \mathrm{~ms}
$$


For a response message of 513 bytes long also transmitted with 115200 bps of baud rate. The return time of data appears equal to:

$$
\text { Treturn }=\frac{513 \cdot 10}{115200} \cong 44,5 \mathrm{~ms}
$$

A fundamental parameter from Imagenex SportScan is the range that it can reach. So, it can be configured for $30,60,90$ or 120 meters from range scale.

The table 2 shows the sample time for this sonar with different configuration range scales, for a speed of sound inside the water of $1450 \mathrm{~m} / \mathrm{s}$.

TABLE II

SAMPLE TIME. IT IS VARIABLE WITH THE RANGE SCALE CHOSEN

\begin{tabular}{|c|c|c|c|c|c|}
\hline Range Scale & 15 & 30 & 60 & 90 & 120 \\
\hline $\mathrm{T}(\mathrm{ms})$ & 67 & 88 & 130 & 171 & 212 \\
\hline
\end{tabular}

The sample time grows with the range scale increase.

Each observation of the sonar is a slice of 500 points that can correspond to a single side (port or starboard) or to the both sides (port and starboard). So, when the side can operation mode chosen is the both sides, each side have only 250 points, but when the single side is used its is represented by 500 points.

The table 3 shows the sonar resolution for the different range scales and for the two possible modes of operation: one or two transducers used.

$$
\text { TABLE III }
$$

RESOLUTION OF ACOUSTIC IMAGE.

\begin{tabular}{|c|c|c|c|c|c|}
\hline Points Range & 15 & 30 & 60 & 90 & 120 \\
\hline 250 & $6 \mathrm{~cm}$ & $12 \mathrm{~cm}$ & $24 \mathrm{~cm}$ & $48 \mathrm{~cm}$ & $96 \mathrm{~cm}$ \\
\hline 500 & $3 \mathrm{~cm}$ & $6 \mathrm{~cm}$ & $12 \mathrm{~cm}$ & $24 \mathrm{~cm}$ & $48 \mathrm{~cm}$ \\
\hline
\end{tabular}

We can see that the resolution decreases with range scale. Can be also seen that when only one sonar side is used, i.e. only starboard or port acquire data of echo strength the resolution is greater then when both sides are used.

The start gain of the echo impulses can be also configured to values between 0 to $40 \mathrm{DB}$.

This sonar also has a GPS interface which provides speed correction and improves the quality of the acoustic image. The GPS interface is not used on this work.

All the characteristics and communication protocol of Imagenex SportScan can be seen on it datasheet [2].

\section{ASV (Autonomous Surface VehicLE) Zarco}

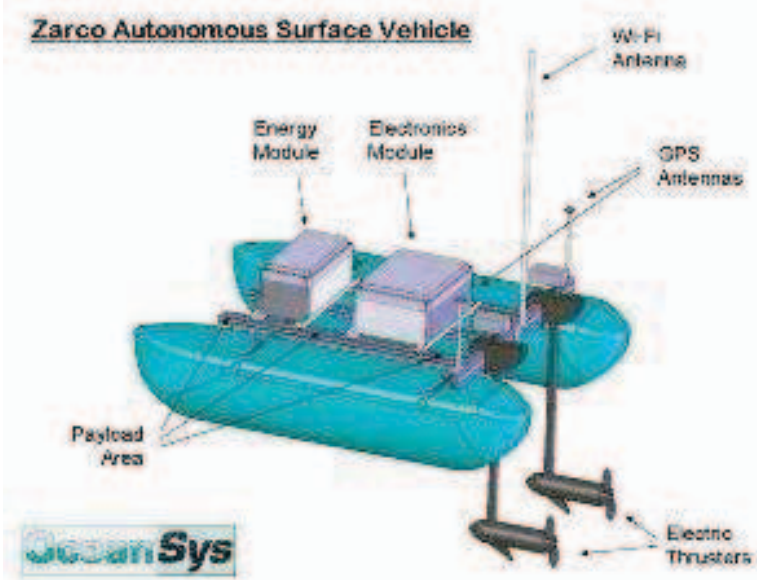

Figure 5 ASV ZARCO that belongs to The OceanSys Group.
The figure 5 shows the ASV components like the two thrusters. The GPS and Wi-Fi antennas and the energy and electronic (PC on-board) modules.

The OceanSys Group has an ASV (Autonomous Surface Vehicle) called ZARCO. This surface vehicle has two electric thrusters that give the capacity to the ASV to move on the horizontal plan.

ZARCO is based on PC stack PC-104 on-board that runs Linux Kernel. The vehicle also carry a router Wi-Fi on-board that with the help of an antenna of higher range provides the communication between the ASV and a laptop located on one reference static point.

The PC on-board of ZARCO also has serial port interface. The serial port communication is made through the RS-232 connector that is used to link the ASV on-board PC to the Imagenex SportScan.

To acquire acoustic images the ASV ZARCO was used and it function is transport the side scan sonar. The figure 6 a) and b) shows the way how the Imagenex SportScan was placed on the ASV ZARCO whose control is made through the Wi-Fi communication and through a joystick to gives him direction.

The ASV ZARCO also has GPS localization in real time.

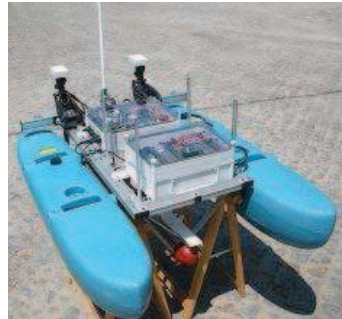

a)

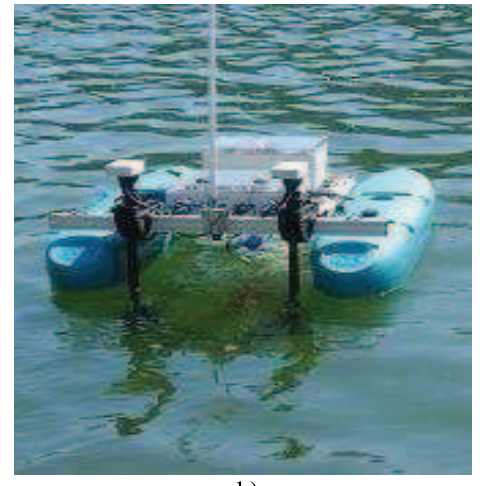

b)
Figure 6 ASV ZARCO. a) Outside of water can be seen the side scan sonar placed under it. b) Inside of water, can be seen that the Imagenex SportScan stay underwater.

\section{COMMUNICATION INTERFACE}

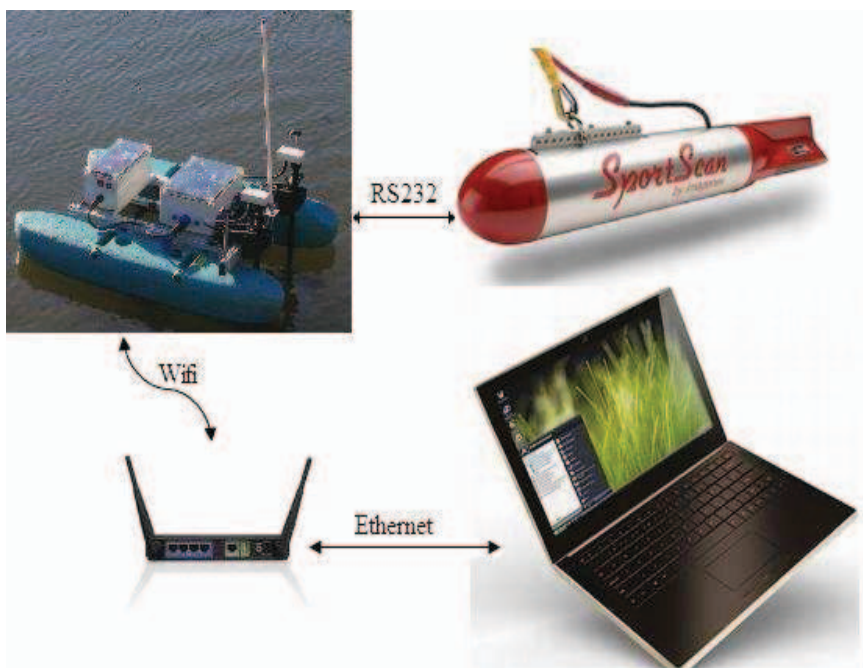

Figure 7 Communication between ImagenexSportcan, Zarco and a static laptop when the marine environment observations can be seen. 
There are a support software for Imagenex SportScan. This software is the Win81ss and it makes the interface between a laptop and the Imagenex SportScan through a physical way like RS-232.

This software provides a serial communication with the sonar and with it is possible see the sonar observation acquired in real time by sonar.

Thus, was intended using the Win81ss interface to see in real time the environment observed during a mission where the sonar is transported by ZARCO.

The figure 7 shows the communication implemented between a static laptop and the ASV ZARCO that transport the sonar.

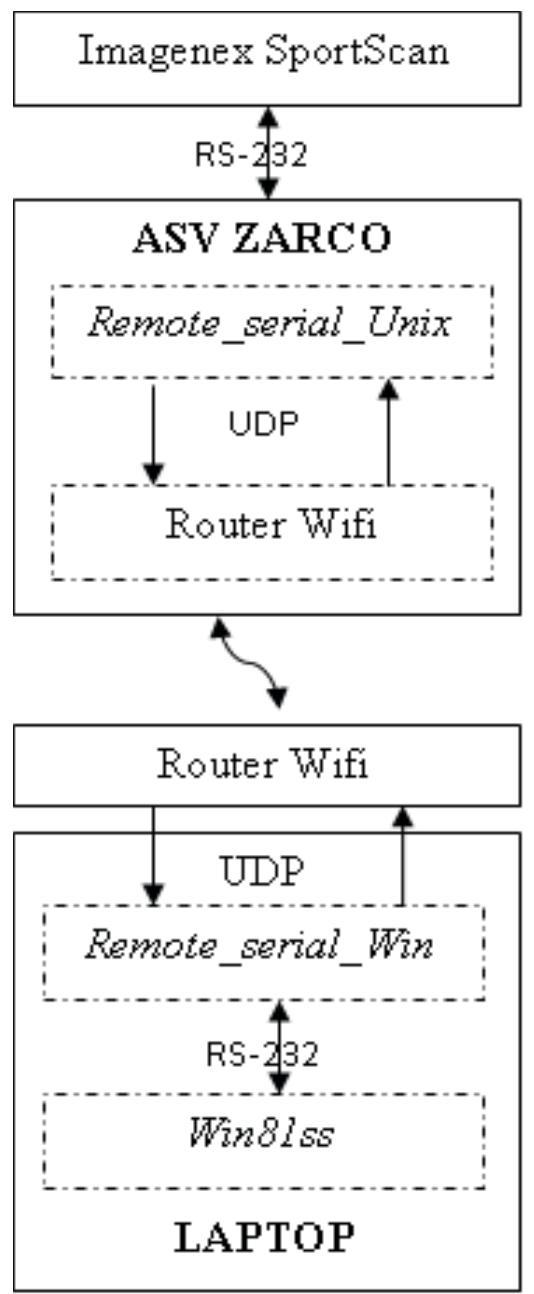

Figure 8 Detailed communication interface between the equipments (ZARCO, Imagenex SportScan and an laptop) used during a mission.

For this communication interface a $\mathrm{C}++$ application (remotesserial_unix for unix and a remote_serial_win for windows) was developed. The interface receives and sends data packages through RS-232 on one side and by UDP on the other side. The complete configuration of the interface of communication is:

-On laptop runs the software Win81ss which provides the seeing of sonar return data.
-The data is exchanged via RS-232 with the own laptop through serial ports and an RS-232 cable.

-The $\mathrm{C}++$ application (remote_seria_winl) exchange data with the Win81ss via serial port and sen $\overline{\mathrm{d}} /$ receive data from the Wi-Fi router through Ethernet cable.

-So, the data is exchanged between the earth and the ZARCO router via $\mathrm{Wi}-\mathrm{Fi}$ and thus the communication between the earth and the ASV PC is guaranteed.

- In the ASV on-board PC the $\mathrm{C}++$ application developed (remote_serial_unix), to linux operative system, runs and make the data exchange with the Imagenex SportScan placed the ASV.

\section{SIDE SCAN OPERATION LIMITS}

Any sonar have a range limit and then it cannot see the environment that is a major distance from this range.

So, when the sonar used is side scan sonar and the need is to follow features like an underwater pipe on the sea floor or a wall, these features cannot for any instant disappear from the range of sonar.

Therefore in this section the side scan operation limits are related.

When the mode of sonar operation is low frequency $(330 \mathrm{Kz})$ the incidence cone of the acoustic impulses is 60 degrees. But, when the high frequency $(800 \mathrm{Khz})$ mode is chosen for sonar acquisition the incidence angle is 30 degrees.

Still about the sonar beam, the middle line of sonar beam makes 20 degrees with the horizontal line. The next figures, figure 9 and figure 10, shows the sonar beam angles decomposition for low and high frequency modes.

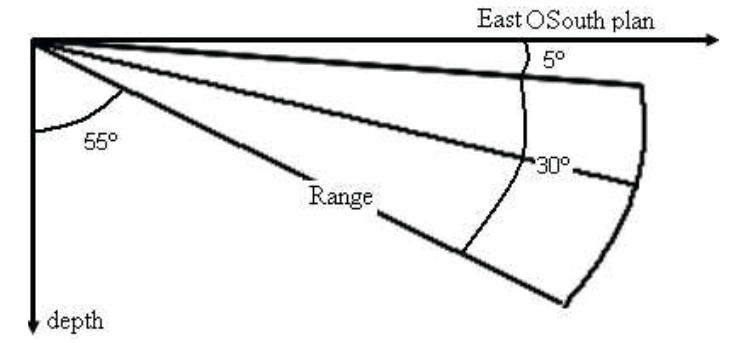

Figure 9 Sonar beam for frequency mode $800 \mathrm{Kz}$, incident angle of $30^{\circ}$.

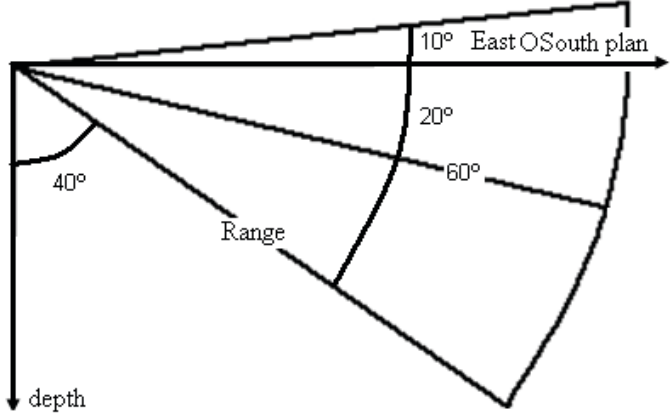

Figure 10 Sonar beam for frequency mode $800 \mathrm{Kz}$, incident angle of $30^{\circ}$.

For operation limits determination of side scan sonar, that was the same limits for the autonomous vehicle that transport the sonar, we need to have in account that on acoustic image the sea floor echo would appear before the feature echo. 
When the feature is a underwater pipe and the goal is follow this pipe, the sonar needs to work inside a area where it can see ever the pipe. So, for the mode of operation of $30^{\circ}$ the operation zone is limited by the follow equations:

$$
\begin{gathered}
h \leq \tan \left(55^{\circ}+30^{\circ}+90^{\circ}\right) \cdot x \cap \\
h \leq \tan \left(55^{\circ}+90^{\circ}\right) \cdot x \cap \\
x^{2}+h^{2} \leq \text { Range } e^{2} \cap \\
h \geq 0 \cap x^{2} \geq\left(\frac{h}{\cos 55^{\circ}}+R\right)^{2}-h^{2},
\end{gathered}
$$

where $h$ is the depth distance between the sonar and the feature. $x$ is equal to the horizontal distance between the sonar localization and feature. Finally, $R$ is underwater pipe radius.

For an angle of incidence beam equal to $60^{\circ}$, the operation limits are given by:

$$
\begin{gathered}
h \leq \tan \left(40^{\circ}+90^{\circ}\right) \cdot x \cap \\
x^{2}+h^{2} \leq \operatorname{Range}^{2} \cap \\
x^{2} \geq\left(\frac{h}{\cos 40^{\circ}}+R\right)^{2}-h^{2}
\end{gathered}
$$

When the feature is a vertical wall, for a incidence angle equal to $30^{\circ}$ the operation zone is equal to:

$$
\begin{gathered}
h \leq \text { Range } \cdot \cos 55^{\circ} \cap x \leq \text { Range } \cdot \cos 5^{\circ} \cap \\
\frac{h}{\cos 55^{\circ}}<\frac{x}{\cos 5^{\circ}}
\end{gathered}
$$

Finally for $60^{\circ}$ :

$$
\begin{gathered}
h \leq \text { Range } \cdot \cos 40^{\circ} \cap x \leq \text { Range } \cap \\
\frac{h}{\cos 40^{\circ}}<x
\end{gathered}
$$

\section{ACOUSTIC IMAGE SEGMENTATION}

The image obtained by the SportScan sonar is a grey scale image (monochromatic image). The image size is equals to $500 \times$ N. Each slice acquired from Imagenex SportScan has 500 points of data. $\mathrm{N}$ corresponds to a number of slices that constitute de image.

To obtain sea floor detection, the sequences of image operations applied are:

-Filter application with the goal of eliminate some noise that can be result from the image acquisition process.

-Detect the minimum value of the picture and thus non-echo zone (black pixels).

-A unimodal threshold is applied with the goal to transform the grey scale image on binary image. Using the minimum value as threshold value the sea floor continues black and the echo from bottom and targets become white, including the surface echo.

-Dilate followed by erode are applied with a structured element with square shape with $3 \times 3$ dimension to eliminate small holes (black pixels) into the bottom echo zone (white pixels).

-Erode followed by dilate are applied with a structured element also with square shape and 10x10 dimension to clear thin lines from surface echo into the non-echo zone (black pixels).

-Horizontal edge detection can be accomplished by applying the square matrix with dimensions $3 \times 3$, Prewitt filter:

$$
\text { Prewitt }=\left[\begin{array}{ccc}
-1 & -1 & -1 \\
0 & 0 & 0 \\
1 & 1 & 1
\end{array}\right]
$$

The convolution of this filter with the image is equal to zero when applied in homogeneous zones and it is different from zero when applied on edge pixels. The convolution result is an image with edges detected and represented by a straight line, figure 4 b) (rigth).

-Finally the Hough transform is employed to obtain line equations.

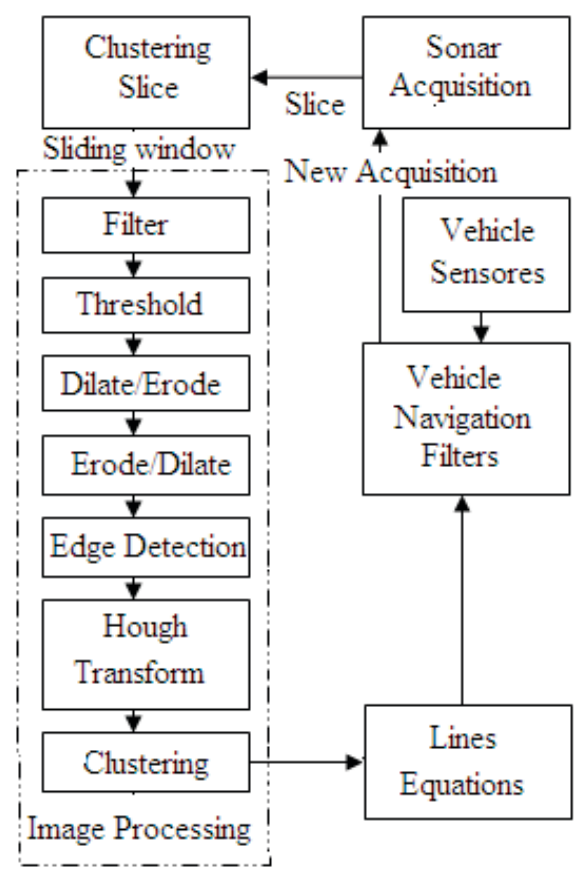

Figure 11 Algorithm to obtain the sea floor equation, starboard and port, and the interaction of lines information with possible vehicle navigation.

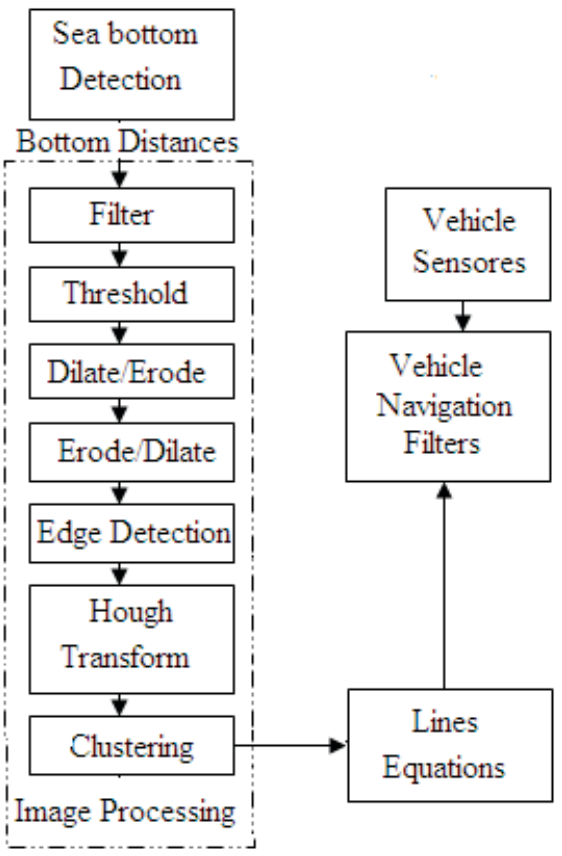

Figure 12 Algorithm to obtain the feature equation, wall or a underwater pipe. The distance to bottom is used in this algorithm. 
To extract feature equations like a pipe or a wall, the procedure is the same with the difference that the threshold value its now equals to maximum value of the picture and thus echo zone (more white pixels).

Thus, when the threshold is applied, the echo zones like the bottom, surface and feature echo appear in the image represented with white pixies. So, the bottom distance obtained with the application of the previous algorithm is now used to discriminate detected lines like the bottom and surface echo.

\section{A. Sliding Window}

Using a sliding window, possible disturbances in image the can be reduced. Each slice acquired by sonar has random noise that is result from the acquisition process. This requires the use of a sliding window to decrease the error measurements due to random noise in sonar data.

Thus, in each sampling instant, the observation will be the new slice acquired by side-scan added to the others slices, according to the sliding window size.

But the sliding window length cannot be very large, otherwise the observations would correspond to features observed at a large distant from the vehicle actual position.

So, a trade-off on sliding window length has to be made and it must be larger as possible.

To determine the sliding window size, the time of acquisition has to be considered. The request frame for sonar data is $B_{\text {req }}$ bytes long. The sonar response length is $B_{\text {res }}$. The acquisition time of data is equal to the time between acoustic impulse transmission and the respective echo arrivals. Considering the data exchange between the vehicle and sonar based on RS-232, the total time acquisition of a slice is given by:

$$
T=B R \cdot B i t s\left(B_{r e q}+B_{r e s}\right)+2 \frac{\text { Range }}{v}
$$

where $B R$ is de baud rate, Bits the number bits per byte, Range the sonar reach in meters and $v$ is the velocity of propagation of the sound in water.

If the goal is to follow a path parallel to the feature, as an underwater pipe on the floor or a wall, the window size will be:

$$
w_{\text {size }}=\frac{D \max }{V \cdot T},
$$

where $D \max$ corresponds the maximum distance between the sonar and the feature on the orthogonal direction. D max will be equal to an image pixel size. $V$ corresponds to vehicle speed on the direction orthogonal to the feature.

\section{B. Real Image Sea Floor Detection}

The extraction of features, which in that case, figure $13 \mathrm{a}$ ), only correspond to sea floor, was made using a sliding window with fixed size of 10 slices, figure $13 \mathrm{~b}$ ) (left).

The extraction algorithm of the sea bottom was applied and validated with the acoustic image from the support $C D$ of Imagenex SportScan.

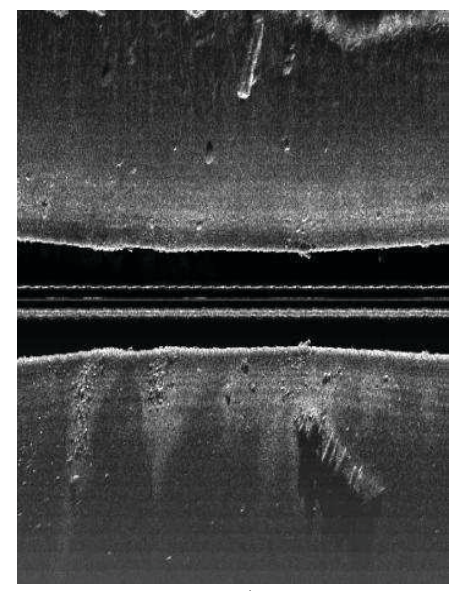

a)

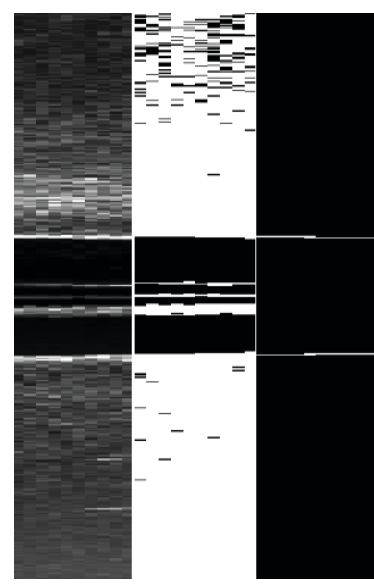

b)
Figure 13 a) Real acoustic image from the support CD of Imagenex SportScan. That image shows the perception of the sea bed. It is possible see when the echo from the floor and surface appears. b) Results. Original sliding window (left). Intermediate result from sliding window segmentation (middle). Final result (right).

\section{Standard Library CImg}

For image treatment, segmentation and features extraction from acoustic images, provided by side scan sonar, the algorithms were implemented through $\mathrm{C}++$ routines. To implement these routines one open source library was used, this library name is $C \operatorname{Img}[17]$.

The CImg is an image treatment library, very useful, that is dedicated to $\mathrm{C}++$ programs. This library has only a header file and it provides a set of functions and classes which allows save, load, manipulate and process images and also show this images.

This library is easily transported by the different operative systems like Unix, Windows or MacOs.

The CImg library has a higher importance in application to image process, it was started in 1999 by David Tschumperle in him doctor graduation thesis [17].

\section{Acoustic Image AcQuired And SEgmentation RESULTS}

In 31 of July of the year 2009, a mission to collect real acoustic images was made.

This mission was made with the Imagenex SportScan placed in ASV ZARCO and the sonar images acquired were seen on-line with the help of the communication interface developed.

This mission took place in the Douro River, near the city of Porto, Portugal, and we obtained various acoustic images. Three examples of acoustic images obtained were presented by figure 14 a) b) and c).

The three images were obtained with the use of the two sonar transducers (starboard and port) and a range scale of 15 meters. Both figures 14 a) and b) were obtained with an incidence angle of $60^{\circ}$ and in both the bottom and the surface (starboard and port) echoes can be seen. The noise inside the no-echo zone is suspended particles inside the water.

The last figure presented, figure $14 \mathrm{c}$ ), was acquired using the high frequency mode and thus with the incident beam angle of $30^{\circ}$. In this image also a wall was caught. As figure 
14 c) has a feature echo it was possible apply the bottom distance algorithm followed by the feature distance extraction algorithm.

The application of the algorithms described, to the sea floor and features extraction, was made on the acoustic images acquired in the river. The distances (starboard and port) in pixies and meters are obtained and are presented with the following graphics (figure 14 to figure 20).

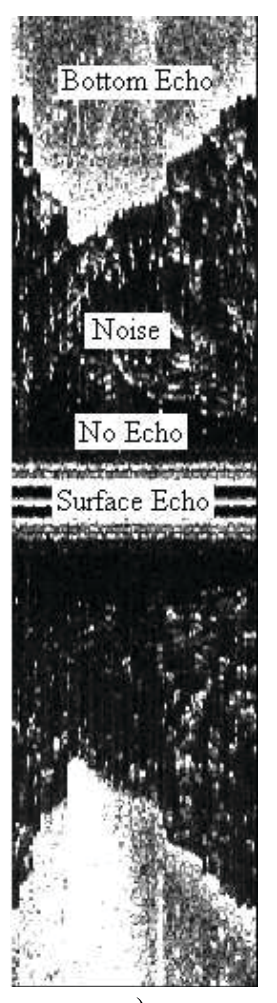

a)

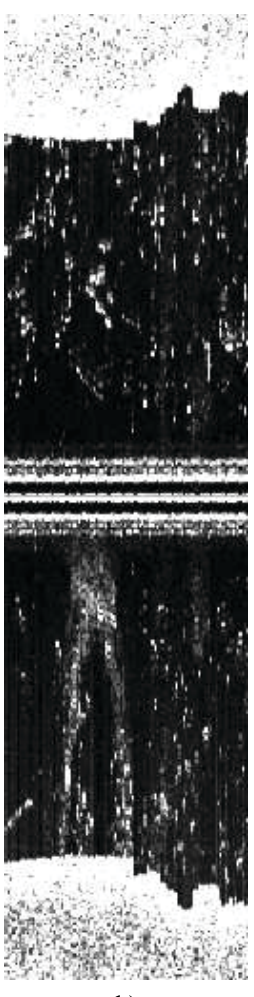

b)

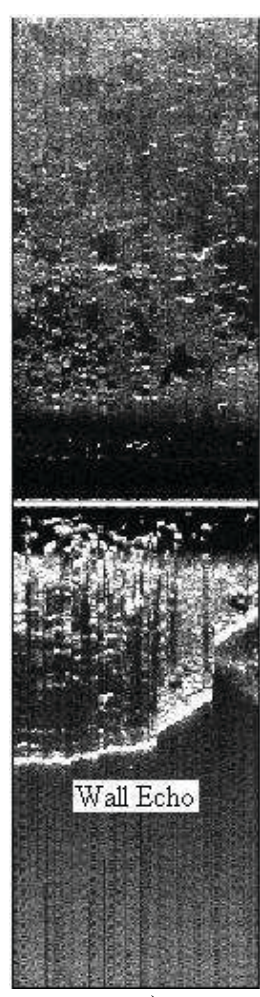

c)
Figure 14 Real acoustic Images obtained during a mission.

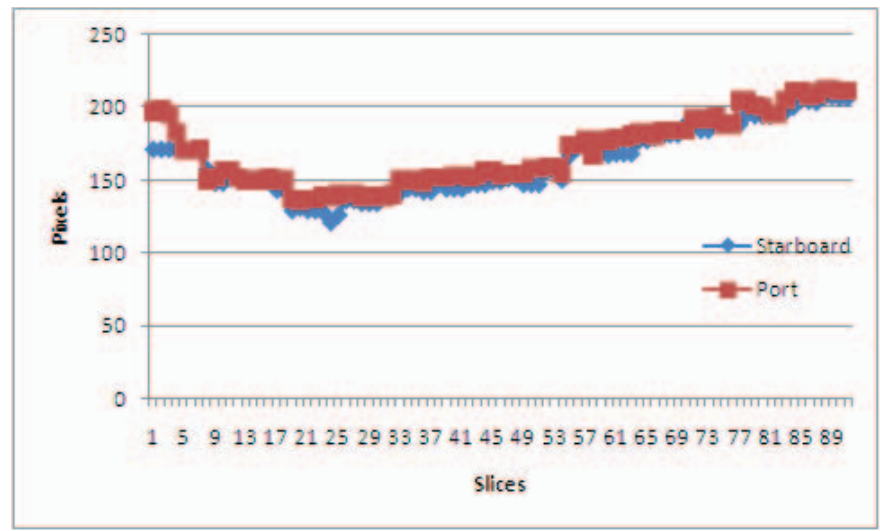

Figure 15 Bottom distance results in pixies applied for figure 14 a).

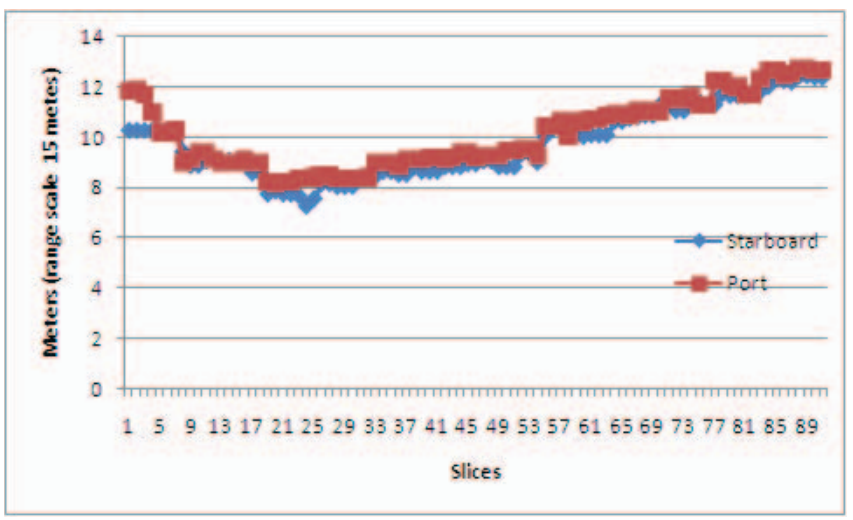

Figure 16 Bottom distance results in meters applied for figure 14 a).

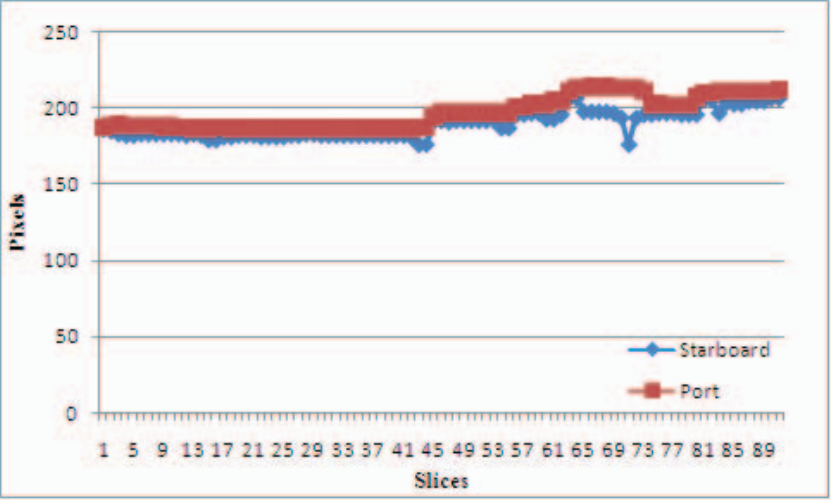

Figure 17 Bottom distance results in pixies applied to figure $14 \mathrm{~b}$ ).

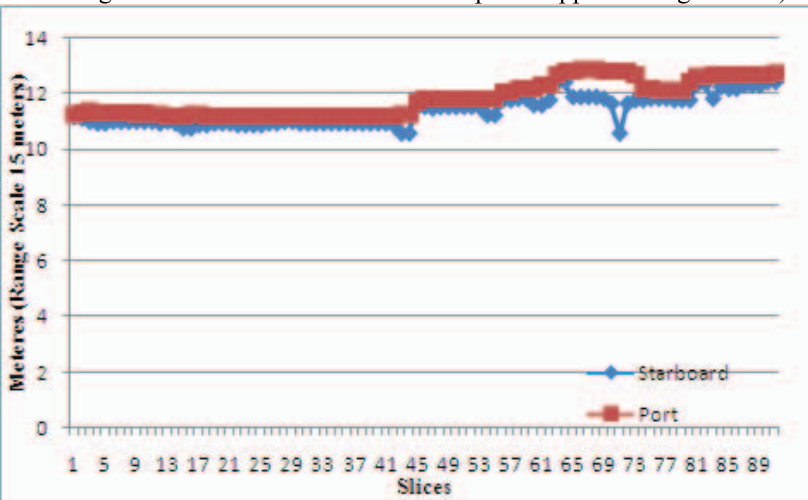

Figure 18 Bottom distance results in metersapplied to figure $14 \mathrm{~b}$ ).

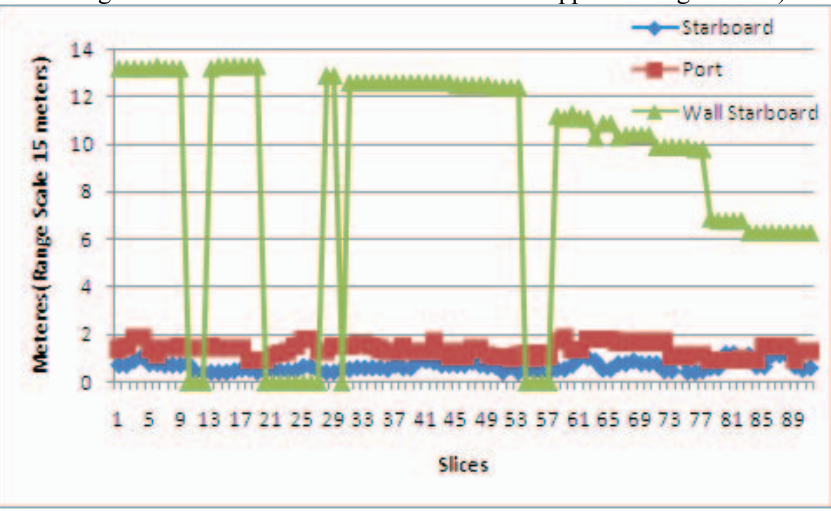

Figure 19 Bottom and starboard wall distance results in pixies applied to figure $14 \mathrm{c}$ ). When the algorithm does not detect the wall distances, the distance given is zero. 


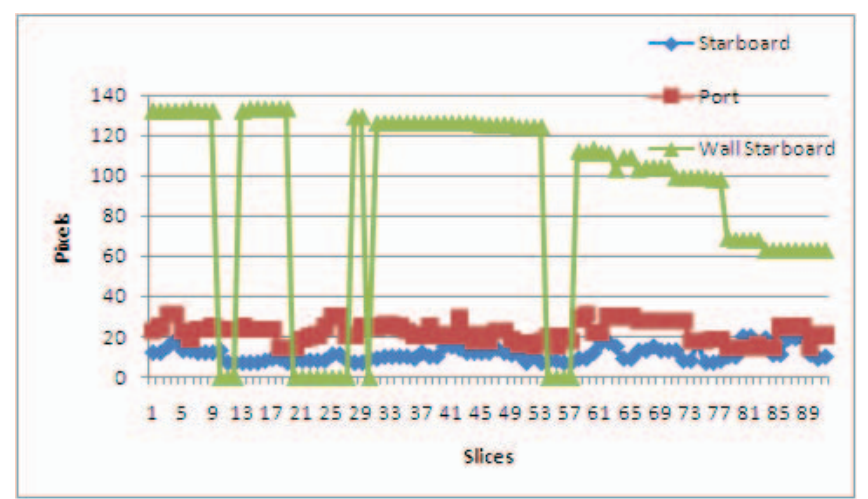

Figure 20 Bottom and starboard wall distance results in meters.

\section{Project Limitations}

The acoustic image is sensible to disturbances caused by the water dirt or by suspended particles inside the water. The result is noise inside the image that can make difficult image processing.

When the goal is, for example, follow a feature like a vertical wall or an underwater pipe that exists inside the marine environment, the sonar need to see for all instants of time the features. Thus, the range scale, the start gain, and the acquisition mode (starboard or port, or both) of the sonar should be dynamic and variable with the goal of never lose the feature in the image and obtain acoustic image of higher quality.

The sonar vision can be used to find lost objects on the sea bottom. This needs human operation. But, when the objective is control the vehicle trajectory based on image seeing provided by sonar, all should be autonomous which requires well structured environments.

Finally, when the sonar is placed on an autonomous vehicle that carried sensors of navigation like bar sensor (that gives the depth), for example, they can be used together with the image segmentation and feature extraction algorithms.

\section{CONCLUSIONS}

The work developed and described here allows the acquisition of real acoustic images and the correspondent features and floor extraction.

The main characteristics from an generic side scan sonar and the typical images obtained by it were studied.

The side scan sonar Imagenex SportScan was used and it fundamental physical, hardware and software characteristics were studied.

Was developed a communication interface that allows see in real time the sonar observations when it is transported by the ASV ZARCO.

Algorithms to features and the distances to the sea floor were implemented in $\mathrm{C}++$, with the CImg library help, and it was validated with the application on real images acquired during a side scan mission.

In future we have the intend to make the extraction algorithms (features and marine bottom extraction) more robustness and flexible.
These algorithms should be applied to more acoustic images for better validation.

\section{REFERENCES}

[1] J. Horgan and D. Toal, "Computer Vision Applications in the Navigation of Unmanned Underwater Vehicles", on "Underwater vehicles", ISBN 978-953-7619-49-7.

[2] Imagenex Sportscan sonar datasheet. It can be found in the web site: http://www.imagenex.com/Downloads/downloads.html. Accessed on: 20 August 2009.

[3] P. E. Hagen,O. Hegrenaes, B. Jalving, O. Midtgaard, M. Wiig and O. K. Hagen, "Making AUVs truly Autonoumous",on "Underwater vehicles", ISBN 978-953-7619-49-7, Publisher: IN-TECH, January 2009.

[4] Michael Scorba, "Simultaneous Localisation and Map Building", Robotic Reacherch Group Department of Engeneering of Oxford, Oxford England, thesis for the Degree of Philosophy, 1997.

[5] D.E. Di Massa and W.K. Stewart Jr., "Terrain-Relative Navigation for Autonomous Underwater Vehicles", Woods Hole Oceanographic Institution.

[6] T. Ruiz, S. de Raucourt, Y. Petillot, and D. M. Lane, "Concurrent Mapping and Localization Using Sidescan Sonar", IEEE Journal of Oceanic Engineering, Vol. 29 No. 2, April 2004.

[7] David Ribas, Pere Ridao, José Neira, Juan D. Tardos, "SLAM using an Imaging Sonar for Partially Structured Underwater Environments", IROS 2006.

[8] Juan D. Tardós, José Neira Dept. Informática e Ingeniería de Sistemas, Universidad de Zaragoza e Paul M. Newman, John J. Leonard MIT Dept. of Ocean Engineering, "Robust Mapping and Localization in Indoor Environments using Sonar Data", 24 July 2002.

[9] T. Ruiz, Y. Petillot and D. M. Lane, "Improved AUV Navigation using Side-scan Sonar", Ocean Systems Laboratory School of Engineering and Physical Sciences Heriot-Watt University, Scotland, UK.

[10] P.Newman H.Durrant-Whyte, "Using Sonar in Terrain-Aided Underwater Navigation", International Conference on Robotics \& Automation Leuven, Belgium, May 1998.

[11] I. T. Ruiz, S. de Raucourt, Y. Petillot, and D. M. Lane, "Concurrent Mapping and Localization Using Sidescan Sonar", IEEE Journal of Oceanic Engineering, Vol. 29 No. 2, April 2004.

[12] I. T. Ruiz, Y. Petillot and D. M. Lane, "Improved AUV Navigation using Side-scan Sonar", Ocean Systems Laboratory School of Engineering and Physical Sciences Heriot-Watt University, Scotland, UK.

[13] "Sidescan Sonar: a general description", It can be found in the web site:http://sub-log.com/sidescan_sonar_general_description. Accessed on: 20 August 2009.

[14] Oddbjorn Bergem, "Bathymetric Navigation of Autonomous Vehicles using a Multibeam Sonar and a Kalman Filter with Relative Measurements Covariance Matrices", University of Trondheim, College of Arts and Science, Department of informatics and computer science, thesis for the Degree of Philosophy, December 1993.

[15] R. C. Gonzalez and R. E. Woods, "Digital Image Processing", ISBN 0201-50803-6, September 1993.

[16] "The OceanSys Group". FEUP-DEEC. It can be found in the web site: http://oceansys.fe.up.pt/. Acessed on: 20 August 2009.

[17] "The CImag Library, C++ Template Image Processing Toolkit". It can be found in the Web site: file://C:/Documents\%20and\%20Settings/Miguel\%20Pinto/Desktop/CI mg-1.3.0/CImg-1.3.0/html $/$ reference/index.html. Accessed on: 20 August 2009. 